\title{
Toward a Configurational Protection Motivation Theory
}

\author{
Yongqiang Sun \\ Wuhan University \\ sunyq@whu.edu.cn
}

\author{
Nan Wang \\ Wuhan University \\ nanwang@,whu.edu.cn
}

\author{
Xiao-Liang Shen \\ Wuhan University \\ xlshen@whu.edu.cn
}

\begin{abstract}
Protection motivation theory (PMT) has been widely used as a theory to explain users' adoption of health information technologies. Prior studies based on PMT tend to treat it as a variance model and explain the parallel and independent effects of its constructs. This theorization neglects the original insights about the sequence of decision making and the interdependencies between PMT constructs. To address both of these two issues, this study proposes and tests a configurational protection motivation theory (CPMT). Specifically, different configurations are identified to reflect the potential sequential effects, substitutive effects, and complementary effects. A survey of 204 mobile health service users in China is conducted to test CPMT and the data analysis results confirm the theoretical expectations. This study can contribute to protection motivation theory and e-health research and suggest practitioners to think in a holistic way during service promotion.
\end{abstract}

\section{Introduction}

Information technologies, specifically e-health technologies, have enabled individuals to better manage their health conditions through a variety of health-related information services including health consulting, health knowledge learning, health status monitoring and maintenance [1, 2]. Despite the advantages of e-health technologies, individuals may still resist to adopt them for some reasons, especially for those populations with low abilities and innovativeness, e.g., elderly [3, 4]. Therefore, a substantial amount of research has engaged in investigating the factors that influence users' adoption or use of e-health technologies [5].

Among the various theories used in prior studies, protection motivation theory (PMT) is the most widely used one. PMT originates from the traditional research on fear appeals and proposes that individuals tend to protect themselves when encountering threats [6]. It is used to explain general health behaviors because health behaviors can be regarded as a solution to cope with the threats of illness [7, 8]. Similarly, regarding the adoption of e-health technologies as an approach to remove illness concerns, PMT provides a solid framework to analyze the factors influencing e-health adoption behaviors [3].

PMT states that individuals' protection motivations are determined by two types of cognitive appraisals: threat appraisals and coping appraisals [6]. Threat appraisals, as primary appraisals, evaluate the severity of the threatening situation (e.g., perceived severity) and the possibility of the occurrence of the threatening situation (e.g., perceived vulnerability). Coping appraisals, as secondary appraisals, assess the issues relevant to the adaptive responses including response efficacy (which refers to the effectiveness of the recommended behavior), self-efficacy (which refers to individual ability to enact the recommended behavior), and response costs (which refer to costs associated with the recommended behavior).

Prior studies based on PMT treat it as a variance model and propose the parallel and independent effects of PMT factors. However, one theoretical foundation of PMT, transaction-based model of stress or coping theory [9], postulates that there is sequence of decision-making from the primary appraisals to the secondary appraisals (e.g., sequential other than parallel effects), and primary and secondary appraisals should be considered as interdependent processes (e.g., interdependent other than independent effects). Ignorance of the sequential and interdependent effects provides partial explanations for the inconsistent findings revealed in prior studies. For example, perceived severity and perceived vulnerability have been found to be significant in some studies but insignificant in other studies [5]. The impacts of response costs are also found to be significant in some studies [10] while insignificant in other studies [11].

The inconsistent findings have the following theoretical implications. First, coping factors may be not considered by users when threats are not perceived, which suggests there is a sequence of decision making, i.e., threat appraisals precede coping appraisals. Taking the decision-making sequence in the theorization can well explain why coping appraisals may have no 
impact on adoption intention [5]. Second, there may be multiple solutions such that the required causal conditions in certain solution can substitute those conditions in other solutions, i.e., substitution effects. Considering the multiple solutions provides another theoretical angle to understand the diversity of the empirical findings [12]. Third, threat appraisals and coping appraisals may affect users' e-health adoption jointly rather than independently such that one factor can exert its impacts only when other factors satisfy certain conditions, i.e., complementary effects. Recognizing the universal interdependencies between constructs can provide a holistic view about the complex phenomenon [12].

The inability of the variance model of PMT in dealing with the sequential and interdependent effects calls for a paradigm shift from the variance model to the configurational model of PMT. Configurational perspective regards outcomes as the results of the configurations of a variety of causal conditions or elements [12]. It can address the sequential issue by treating threat appraisals as the pre-conditions for coping appraisals to work, such that when threat appraisals are absent, whether there are coping appraisals does not matter. It can address the interdependent issue by considering e-health adoption as the joint results of all the PMT factors such that these factors interdependently rather than independently exert their impacts. Further, based on the principle of equifinality, configurational perspective also suggests there should be multiple solutions which can lead to the same outcome [12]. Given the match between the configurational perspective and the research gaps in variance-based PMT, this study tries to propose a configurational protection motivation theory (CPMT) to advance the theoretical understanding on PMT. Specifically, CPMT will extend PMT by shedding light on the sequential effects, substitution effects, and complementary effects of PMT factors.

\section{Theoretical background}

\subsection{Protection motivation theory (PMT)}

PMT can be regarded as a theory based on the coping theory [9] and expectancy-value theory [13]. According to coping theory, the key statements of PMT classify two types of cognitive appraisals: threat appraisals and coping appraisals [6]. According to PMT, when encountering a threat, individuals will firstly evaluate the extent to which the threat is (i.e., threat appraisals) and then evaluate the extent to which the recommended behavior (e.g., e-health adoption) can cope with the threat (i.e., coping appraisals). Specifically, threat appraisals consist of perceived severity and perceived vulnerability according to expectancy - value theory, where perceived severity captures the losses (value) caused by not adopting the recommended behavior while perceived vulnerability reflects the possibility (expectancy) that individuals experience harm. Coping appraisals consist of response efficacy, self-efficacy and response costs. Specifically, response efficacy refers to the extent to which adopting the recommended behavior can avoid the potential harm; self-efficacy, adopting from the social cognitive theory [14], refers to the degree to which individuals have the abilities to enact the recommended behavior; response costs refer to the costs associated with the recommended behavior. Although rewards for not adopting the recommended behavior are included in the revised version of PMT [15], the most widely used five factors are perceived severity, perceived vulnerability, response efficacy, self-efficacy, and response costs. According to the variance perspective of PMT, two factors related to threat appraisals (e.g., perceived severity and perceived vulnerability) and two factors associated with coping appraisals (e.g., response efficacy and self efficacy) are proposed to positively affect protection motivation while response costs are proposed to negatively influence protection motivation.

In information systems (IS) research, PMT has been widely used in two major research areas: e-health [5] and IS security [16]. In e-health research, adoption of e-health technologies is regarded as a protection behavior, so threat appraisals are related to the threats of disease for not adopting e-health technologies and coping appraisals are about benefits and costs for adopting e-health technologies and users' abilities to adopt these technologies [3]. In IS security research, IS security behaviors are regarded as a protection behavior, and threat appraisals are about the threats induced by those behaviors violating security policies while coping appraisals are relevant to the consequences of IS security behaviors $[17,18]$. Despite the differences in e-health and IS security behaviors, the underlying mechanisms to explain these two types of behavior in terms of PMT are quite similar.

However, prior studies on PMT reveal inconsistent findings about the impacts of PMT factors. As shown in the meta-analysis of the e-health literature [5] and IS security literature [16], PMT factors are found to have significant effects in certain studies while insignificant effects in other studies. Specifically, Boss et al. [19] found the significant effect of only perceived severity while other PMT factors had insignificant effects; Johnston et al. [20] found that only perceived vulnerability had significant effects on protective 
intention while other PMT factors did not; Menard et al. [21] only confirmed the significant relationship between response efficacy and behavioral intention; self efficacy and response costs were also found to be significant in certain studies [22].

There are two possible explanations for the inconsistent findings. First, it is not so necessary to have all the PMT factors satisfy the requirements such that it is possible that users still would like to enact protective behaviors even if certain criteria are not met. This possible explanation leads to potential multiple solutions rather than a single solution for individuals' decision-making on protective behaviors. For example, perceived severity may in some extent substitute the role of perceived vulnerability, such that the existence of only one of the two factors (e.g., perceived severity and perceived vulnerability) is adequate to trigger fear (i.e., substitution effect). Second, PMT factors may function interdependently rather than independently, which calls for understanding the impacts of a certain PMT factors by simultaneously considering the conditions of other PMT factors. Specifically, coping appraisals may become not so important when perceived threat is low because the decision sequence of threat appraisals and coping appraisals (e.g., sequential effect). Individuals may still tend to engage in protective behaviors even if response costs are high given that response efficacy is high enough to surpass response costs (e.g., complementary effect). The inability of variance model to deal with the issues of multiple solutions and multiple interdependencies calls for understanding PMT from a new theoretical perspective - configurational perspective.

\subsection{Configurational perspective}

Unlike the variance perspective of theory building which is based on co-variances between different factors, the configurational perspective interprets cause-effect relationships based on typologies [12]. Typologies have advantages in theory building because of their abilities to deal with multidimensional and complex research problems in a holistic view. Table 1 summarizes the key distinctions between variance and configurational perspectives of theory building from three aspects: complexity, equifinality, and asymmetry.

Table 1. Variance vs. configurational perspectives

\begin{tabular}{lcc}
\hline & Variance & Configurational \\
& perspective & perspective \\
\hline Complexity & Partial view & Holistic view \\
Equifinality & Single solution & Multiple solutions \\
Asymmetry & Symmetric & Asymmetric \\
\hline
\end{tabular}

As to complexity, variance perspective may focus on the independent effects of different factors. Although variance perspective also can deal with the interdependencies between limited factors through moderation effects, it may only capture the interdependencies between two (two-way interaction) or three (three-way interaction) factors. It is difficult for variance models to theorize and test the interdependencies between more than three factors [12] The independent effects or limited interdependent effects revealed by variance models provide only a partial view of the whole picture. In contrast, configurational perspective considers outcomes as the results of the configurations of a variety of causal conditions and stresses on the joint effects of these causal conditions. The key assumption of the configurational perspective relies on that each causal condition cannot lead to the outcome separately and the existence of other causal conditions gives meanings to the focal causal condition [12]. Therefore, configurational perspective views the phenomenon in a holistic way.

As to equifinality, variance perspective holds that there is only one solution for a research model such that one independent variable may have significant or insignificant effects on dependent variables but not the both. The variance model provides consistent practical suggestions for handling the independent variables, i.e. enhancing the variable if it has a positive influence but reducing it if it has a negative influence. However, configurational perspective regards that there are multiple solutions which can reach the same outcome (i.e., equifinality) [12]. Thus, it is possible that one causal condition is present in one configuration while absent in another configuration. Not the presence or absence of one single condition matters but the configurations of a set of conditions matter.

As to causal asymmetry, variance perspective believes that the underlying mechanisms to explain the impacts of independent variables on dependent variables are symmetric. Specifically, if one independent variable is proposed to positively affect a dependent variable, it assumes that: the higher the independent variable, the higher the dependent variable; the lower the independent variable, the lower the dependent variable. The causes for high or low outcome variables are consistent. However, configurational perspective postulates that the causes leading to high outcomes are quite different from those leading to low outcomes [12]. High and low outcomes can be obtained based on different configurations of causal conditions, so the underlying mechanisms are asymmetric rather than symmetric. This suggests that users may adopt or dis-adopt a technology for different reasons. 
Due to the advantages in dealing with complex and asymmetric mechanisms, configurational perspective has become an important angel to rethink about IS research. For example, El Sawy et al. [23] firstly suggest to view digital ecodynamics from a configurational perspective. From this perspective, Park et al. [24] empirically analyze the impacts of business intelligence and communication technologies on organizational agility. Within our research context, regarding the theoretical tension to address the issues of multiple solutions and interdependencies between PMT factors, this study tries to propose CPMT which will analyze the sequential effects, substitutive effects, and complementary effects that are not well understood based on the variance model of PMT.

\section{Hypothesis development}

\subsection{Sequential effect}

PMT suggests that threat appraisals are primary appraisals while coping appraisals are secondary appraisals [6]. According to coping theory, there should be a decision sequence for these two types of cognitive appraisals, such that threat appraisals precede coping appraisals [9]. To capture the sequence of decision-making, we propose that protective motivation is driven by a two-stage decision-making process [25]. Figure 1 shows the decision tree for protective behaviors.

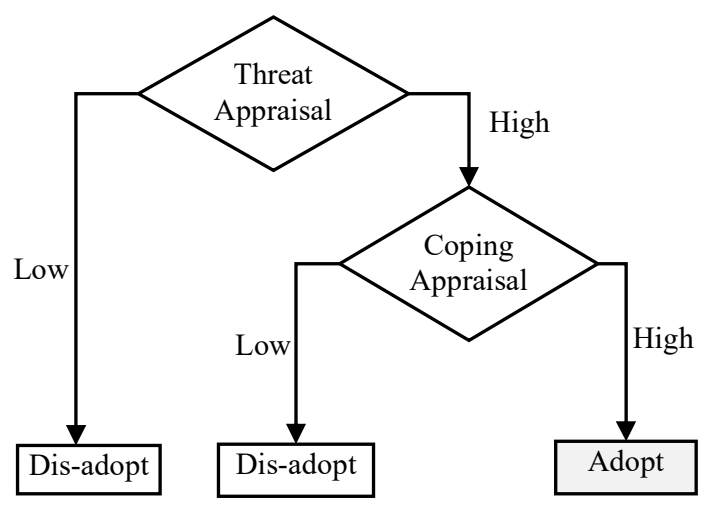

Figure 1. Decision tree for protective behaviors

Specifically, users will firstly evaluate the severity and vulnerability of suffering the health-related problems and decide to dis-adopt the e-health technology when perceived severity and perceived vulnerability are low, because it is not so necessary to adopt the e-health technology (i.e., lack of need). When users regard the severity or vulnerability as high, their fear will be triggered and they will be motivated to make coping appraisals [25]. When users believe that adopting the e-health technology can well solve the problems, with acceptable costs and they have the abilities to use the technology, they will decide to adopt the technology. In contrast, they will dis-adopt the technology when coping appraisals are not satisfied

The sequential decision-making process of protective motivation has two theoretical implications. First, threat appraisals and coping appraisals are interdependent. Cismaru et al. [26] summarize the potential interaction effects among threat appraisals and coping appraisals found in prior studies including the interaction effects between perceived vulnerability and response efficacy, between perceived vulnerability and self-efficacy, and between perceived severity and response efficacy. Liang et al. also proposes [25] and empirically confirms [27] the interaction effect between perceived threat and perceived avoidability. Second, threat appraisals have priority over coping appraisals [18]. That means that when threat appraisals are low, users will decide to dis-adopt the technology regardless whether coping appraisals are high or low. Therefore, the configurations for adoption and disadoption should be different. Users may adopt the technology when both threat appraisals and coping appraisals are high but dis-adopt it as long as one of the two appraisals is low. Therefore, we propose the hypothesis related to the sequential effect as follows:

H1 (sequential effect hypothesis): Adoption can be caused by the configurations with the presence of both threat appraisals and coping appraisals; Disadoption can be caused by the configurations with the absence of threat appraisals, or with the presence of threat appraisals but the absence of coping appraisals.

\subsection{Substitutive effect}

According to the principle of equifinality or neutral permutation, configurational models can provide multiple solutions leading to outcomes while not all the causal conditions are required for all the solutions. If there are two configurations sharing the same causal conditions A (A can be one causal condition or a set of causal conditions), one configuration with a set of causal conditions $\mathrm{B}$ besides $\mathrm{A}$ and the other configuration with a set of causal conditions $\mathrm{C}$ besides $\mathrm{A}$, then $\mathrm{B}$ and $\mathrm{C}$ can be regarded as substitutive conditions [12]. It means that $\mathrm{B}$ and $\mathrm{C}$ play similar roles.

In CPMT, we focus on the substitutive effect between perceived severity and perceived vulnerability. To trigger fear, it is not so necessary to have both high perceived severity and perceived vulnerability, although the fear should be highest when both perceived severity and vulnerability are high. Fear may be triggered when only one of the two threat appraisals 
(e.g., perceived severity and perceived vulnerability) is high enough. It is to say the effect of perceived severity may be partially substituted by perceived vulnerability, vice versa. In prior studies, Das et al. [28] and Pechmann et al. [29] have found that perceived severity has a stronger impact on intention when perceived vulnerability is low, providing supports to the potential substitutive effect between perceived severity and perceived vulnerability. Therefore, CPMT proposes that there may be two configurations: one configuration with only the presence of perceived severity and the other with only the presence of perceived vulnerability. That is:

H2 (substitutive effect hypothesis): As to the configurations for adoption, at least one condition of threat appraisals (e.g., perceived vulnerability and perceived severity) should be present.

\subsection{Complementary effect}

Unlike variance model of PMT which asks for satisfying all the requirements (e.g., high perceived severity, perceived vulnerability, response efficacy, and self-efficacy and low response costs), CPMT suggests that some causal conditions may be not as expected if there are other causal conditions which can compensate the negative consequences. For example, users may still adopt the e-health technology even when responses costs are high or when self-efficacy is low. CPMT proposes the complementary effects between PMT factors to address these unexpected results.

Specifically, when making decision about whether to adopt the e-health technology, users rely on the tradeoff between the benefits and the costs induced by technology adoption rather than solely benefits or costs. The tradeoff process indicates the interdependence between benefits and costs. Thus, users may still enact adoption behavior when response costs are high if response efficacy is high enough such that response efficacy exceed response costs making the adoption behavior to be worthy (e.g., the complementary effect between response efficacy and response costs). This complementary effect can be supported by empirical evidences from Krishnamurthy et al. [30].

Another complementary effect is related to the interdependence between response efficacy and selfefficacy. Self-efficacy may be not a necessary condition for technology adoption if the technology is regarded to be very useful. Users may solve the problem of self-inefficacy through learning or indirect use which refers to that designated users may not interact directly with the technology but receive and use the information produced by the technology via intermediary users $[31,32]$. It is common that elderly users may ask their adult children who are familiar with information technologies to use e-health technologies on behalf of them. Thus, users may still adopt the e-health technology even if the self-efficacy is low because of the complementary effect between response efficacy and self-efficacy. Therefore, we propose that:

H3 (complementary effect hypothesis): As to the configurations for adoption, response costs can be present when response efficacy is present; self-efficacy can be absent when response efficacy is present.

\section{Research method}

\subsection{Research setting}

A field survey in North China was conducted to test the proposed hypotheses. We collaborated with one of the biggest companies which provided mobile health services for the elderly. The mobile health services were provided by the target company in collaboration with the local government. These services included consulting and assistance center, customized terminal and remote positioning services. When conducting the survey, the mobile health services was just released to the market. Respondents of the survey were recruited from the potential consumers during the service promotion process.

\subsection{Measures}

The measures for all the PMT factors were adapted from prior studies (see Appendix). Specifically, intention to adopt, perceived severity, perceived vulnerability, response efficacy and self-efficacy were measured with the items adapted from Johnston and Warkentin [18] and adjusted to align with research context of mobile health services. Response costs were measured with the items adapted from Lee and Larsen [33]. Seven-point Likert scales were used for all items. The items were translated into Chinese through a backtranslation approach [34].

\subsection{Data collection procedure}

The target company helped to collect the data through community service centers which had good relationships with the target company. The respondents were recruited when the company provided routine training for its customers. During the training, the company firstly introduced the functions of the mobile health services and then potential customers were 
asked to participate in the survey voluntarily. To encourage participation, some daily necessities (e.g., eggs) were provided as the incentives because these incentives were consistent with their needs.

After removing the incomplete cases and outliers, 204 valid responses were obtained. Among these subjects, female subjects occupy $46.6 \%$, and over $80 \%$ of the subjects are over 40 years of age. The education level for $52.9 \%$ of the subjects is high school or below; approximately $51.5 \%$ have fewer than two years of computer experience, and about $70 \%$ of subjects have more than two years of mobile device usage experience.

\section{Data analysis}

Partial Least Squares (PLS), specifically SmartPLS, was used to evaluate the measurement model while fuzzy-set qualitative comparative analysis (fsQCA) was used to test the hypotheses. As a structural equation modeling technique, PLS can provide a systematic estimation of the loadings of the indicators on constructs. Compared with covariance-based techniques, PLS is more suitable for relatively small samples [35]. QCA has been widely used to identify the configurations of causal conditions, and fsQCA specifically can deal with the continuous variables [36].

\subsection{Measurement model}

Measurement model can be evaluated by checking the reliability and validity of the constructs. Reliability can be assessed according to the values of composite reliability (CR) and average variance extracted (AVE), where the threshold values for CR and AVE are 0.7 and 0.5 respectively $[37,38]$. As shown in Table 2 , the CRs for all the constructs were greater than 0.8 and the AVEs for all the constructs were greater than 0.5 , indicating that these constructs were with good reliabilities.

Validity includes convergent validity and discriminant validity. Convergent validity is assessed through the item loadings on the respective constructs such that the higher the loading, the better the convergent validity $[39,40]$. As shown in Table 2 , the item loadings for all the constructs were above 0.7 , suggesting that these constructs had good convergent validities. Discriminant validity can be assessed by checking whether all the correlations relevant to one construct are smaller than the square root of its AVE [37]. As shown in Table 2, the square roots of AVEs were higher than the correlations, suggesting good discriminant validities of these constructs.

\subsection{Hypothesis testing}

After the measurement model was ensured, the items of each construct were packaged into one value using their mean value. The raw continuous values were further transformed into membership scores according to the calibration process. Based on the membership scores, a truth table was generated and refined by setting frequency cutoff and consistency cutoff. Finally, the complex solution, parsimonious solution, and intermediate solution were reported based on counterfactual analysis (see more technical details in Ragin [36]). In terms of these solutions, the configurations for adoption and dis-adoption were identified as shown in Table 3 and Table 4. Black circles ("•") indicated the presence of a condition, while circles with a cross-out ("®") indicated the absence of a condition. Core conditions were captured through large circles, while peripheral conditions were reflected through small circles.

Specifically, there were four configurations or solutions for adoption. All these four solutions asked for the co-presence of threat appraisals and coping appraisals. For threat appraisals, at least perceived vulnerability or perceived severity should be present (see the later discussion on substitutive effect). For coping appraisals, response efficacy should be present while response costs or self-efficacy might be present or absent (see the later discussion on complementary effect). In contrast, there were six solutions for disadoption. Among these six solutions, both perceived vulnerability and perceived severity were absent in the first three solutions. Although either perceived vulnerability or perceived severity was present in the last three solutions, response efficacy was absent. These results confirmed the theoretical expectations about the sequential effect, e.g., adoption was caused by the co-presence of threat and coping appraisals while dis-adoption was caused by the absence of either threat or coping appraisals. Thus, H1 was supported.

Solution 3 and solution 4 for adoption provided empirical support for the hypothesis on substitutive effect (i.e., H2). Specifically, solution 3 showed that adoption could be caused when only perceived vulnerability was present, while solution 4 reflected that adoption could be caused when only perceived severity was present.

Solution 1 for adoption showed that when response efficacy was present, whether there was self-efficacy did not matter. This suggested that users might adopt the technology even when self-efficacy was absent if response efficacy was high enough, confirming the complementary effect between response efficacy and self-efficacy. Solution 2 for adoption showed that when response efficacy was present, whether there 
were response costs did not matter, indicating that users might adopt the technology even when response

Table 2. Correlation, reliability and validity

\begin{tabular}{cccccccccccc}
\hline & Mean & SD & AVE & CR & Loading & AI & PS & PV & RC & RESP & SEFC \\
\hline AI & 3.77 & 0.75 & 0.76 & 0.90 & {$[0.77,0.91]$} & $\mathbf{0 . 8 6}$ & & & & & \\
PS & 4.21 & 0.70 & 0.72 & 0.89 & {$[0.82,0.89]$} & 0.26 & $\mathbf{0 . 8 5}$ & & & & \\
PV & 3.74 & 0.92 & 0.73 & 0.89 & {$[0.84,0.86]$} & 0.36 & 0.59 & $\mathbf{0 . 8 5}$ & & & \\
RC & 2.72 & 0.78 & 0.59 & 0.81 & {$[0.70,0.81]$} & -0.34 & -0.14 & -0.16 & $\mathbf{0 . 7 7}$ & & \\
RESP & 3.95 & 0.71 & 0.72 & 0.89 & {$[0.83,0.86]$} & 0.49 & 0.31 & 0.44 & -0.32 & $\mathbf{0 . 8 5}$ & \\
SEFC & 3.68 & 0.75 & 0.73 & 0.899 & {$[0.83,0.87]$} & 0.43 & 0.15 & 0.16 & -0.36 & 0.22 & $\mathbf{0 . 8 5}$ \\
\hline
\end{tabular}

Note: $\mathrm{AI}=$ Intention to adopt, $\mathrm{PS}=$ Perceived severity, $\mathrm{PV}=$ Perceived vulnerability, $\mathrm{RC}=$ Response costs, $\mathrm{RESP}=\mathrm{Response}$ efficacy, SEFC = Self-efficacy. AVE denotes average variance extracted, while CR denotes composite reliability. The bold numbers on the diagonal are the square roots of AVEs.

Table 3. Configurations for adoption

\begin{tabular}{|c|c|c|c|c|}
\hline & 1 & 2 & 3 & 4 \\
\hline Perceived Vulnerability & 0 & $\bullet$ & 0 & \\
\hline Perceived Severity & $\bullet$ & 0 & & 0 \\
\hline Response Efficacy & 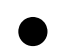 & 0 & 0 & 0 \\
\hline Response Costs & $\otimes$ & & $\otimes$ & $\otimes$ \\
\hline Self-Efficacy & & 0 & $\bullet$ & 0 \\
\hline Raw coverage & 0.46 & 0.44 & 0.46 & 0.43 \\
\hline Unique coverage & 0.07 & 0.05 & 0.06 & 0.03 \\
\hline Consistency & 0.90 & 0.91 & 0.92 & 0.91 \\
\hline Solution coverage & \multicolumn{4}{|c|}{0.60} \\
\hline Solution consistency & \multicolumn{4}{|c|}{0.87} \\
\hline
\end{tabular}

Table 4. Configurations for dis-adoption

\begin{tabular}{|c|c|c|c|c|c|c|}
\hline & 1 & 2 & 3 & 4 & 5 & 6 \\
\hline Perceived Vulnerability & $\otimes$ & $\otimes$ & $\otimes$ & & $\bullet$ & \\
\hline Perceived Severity & $\otimes$ & $\otimes$ & $\otimes$ & $\otimes$ & $\bullet$ & $\bullet$ \\
\hline Response Efficacy & $\otimes$ & $\otimes$ & $\bullet$ & $\otimes$ & $\otimes$ & $\otimes$ \\
\hline Response Costs & & $\bullet$ & $\otimes$ & $\bullet$ & $\otimes$ & $\otimes$ \\
\hline Self-Efficacy & $\otimes$ & & $\bullet$ & $\otimes$ & & - \\
\hline Raw coverage & 0.34 & 0.34 & 0.20 & 0.34 & 0.29 & 0.30 \\
\hline Unique coverage & 0.04 & 0.03 & 0.03 & 0.03 & 0.02 & 0.01 \\
\hline Consistency & 0.91 & 0.90 & 0.79 & 0.86 & 0.79 & 0.79 \\
\hline Solution coverage & \multicolumn{6}{|c|}{0.62} \\
\hline Solution consistency & \multicolumn{6}{|c|}{0.75} \\
\hline
\end{tabular}

costs were high. It was consistent with the arguments about the complementary effect between response efficacy and response costs. Thus, the complementary effect hypothesis (i.e., H3) was supported.

\section{Discussion}

\subsection{Theoretical implications}


This study can contribute to protection motivation theory (PMT) and e-health literature in several ways. First, beyond the variance perspective of PMT (VPMT), this study proposes a configurational PMT (CPMT) to theorize the multiple interdependencies among PMT factors in a holistic view. CPMT has the advantages over VPMT in providing multiple solutions, capturing joint effects, and revealing asymmetric mechanisms. CPMT can better explain the inconsistent findings existing in prior literature by interpreting these findings as different solutions which can reach the same outcome [16]. CPMT can respond to the call for exploring the potential two-way, three-way, and even four-way interaction effects among PMT factors [26]. CPMT also sheds light on the different mechanisms driving technology adoption versus dis-adoption or continuance vs. discontinuance [41]. Viewing PMT from a configurational perspective can solve a series of theoretical problems which cannot be dealt with by the variance models.

Second, this study figures out the decision-making sequence of protective behavior and interprets the sequential effect through the configurations. Although original PMT or coping theory implicitly assumes that threat appraisals precede coping appraisals, a lot of empirical studies based on PMT tend to treat threat and coping appraisals in parallel. To include the decision sequence into the theorization, this study depicts the decision-making process using a decision tree. According to this decision tree, we further identify the different configurations for adoption and dis-adoption based on the different paths of the decision tree. Specifically, we theorize that adoption behavior can be caused by the co-presence of threat and coping appraisals while dis-adoption behavior can be caused when at least one of the two types of cognitive appraisals is absent. This not only provides theoretical explanations about the sequential effect but also offers a new approach to capture the sequential effect.

Third, this study points out the substitutive effect between perceived vulnerability and perceived severity. VPMT suggests to maximize the variables with positive effects while minimize the variables with negative effects. However, CPMT indicates that it is not so necessary to satisfy all the criteria. Fear can be triggered when only perceived vulnerability or perceived severity is high enough, so the effect of perceived vulnerability can be substituted by perceived severity, vice versa. This explains why these two threat appraisals are not consistently found to be significant across studies.

Finally, this study reveals the complementary effects between response efficacy and response costs and between response efficacy and self-efficacy.
VPMT suggests that users will dis-adopt a technology if response costs are high, while it contradicts with the reality that users may still adopt the technology if it is very effective. Given that the benefit-cost tradeoff rather than solely the benefits or costs matter, it is necessary to consider the joint effects of benefits and costs. Similarly, users may tend to learn to enhance their abilities or use the technology in an indirect way, so they may still adopt the technology even if selfefficacy is low. VPMT sheds light on these complementary effects through the configurations of the factors related to coping appraisals.

\subsection{Practical implications}

CPMT can provide suggestions to practitioners too. First, CPMT suggests that e-health service providers should consider the configurations of PMT factors in a holistic view during the service promotion process. For example, they should not formulate promotion strategies solely through threat appeals or coping appeals but the coherence between these appeals. Second, the substitution effect between perceived severity and perceived vulnerability suggests that it is not so necessary to stress on both severity and vulnerability when designing threat appeals. The design of threat appeals should focus on one side of threat appeals according to consumers' preferences to reduce design costs. Third, the complementary effect between response efficacy and response costs suggest that service providers do not need set low price if their services are good enough. The complementary effect between response efficacy and self-efficacy suggests that service providers do not need to be worried about the self-inefficacy of users if their services are good enough, because users may try to enhance their abilities through learning or use it indirectly.

\subsection{Limitations and future research}

This study has several limitations and the findings derived from this study should be used with caution. First, this study is conducted in the research context of mobile health services in China. Whether the findings can be generalized to other services (e.g., IS security) and other countries should be further examined in future research. Second, there are several different versions of PMT (e.g., four-dimension version without response costs and six-dimension version with rewards) and this study is based on the five-dimension version. More empirical work should be done to check the robustness of the conclusion. Third, PMT can be extended by considering other factors such as social influence, so it is interesting to identify the 
configurations according to the extended PMT framework in future research.

\section{Acknowledgements}

The work described in this paper was partially supported by the National Natural Science Foundation of China $(71974148,71904149)$ and the Humanities and Social Sciences Foundation of the Ministry of Education, China (16YJC870011, 17YJC630157).

\section{Appendix. Measures}

Intention to Adopt (AI): Johnston and Warkentin [18] AI1. I intend to use mobile health services in the next 3 months.

AI2. I predict I will use mobile health service in the next 3 months.

AI3. I plan to use mobile health services in the next 3 months.

Perceived Vulnerability (PV): Johnston and Warkentin [18]

PV1. I am at risk for suffering the stated problems.

PV2. It is likely that I will suffer the stated problems.

PV3. It is possible for me to suffer the stated problems.

Perceived Severity (PS): Johnston and Warkentin [18] PS1. If I suffered the stated problems, it would be severe.

PS2. If I suffered the stated problems, it would be serious.

PS3. If I suffered the stated problems, it would be significant.

Response Efficacy (RE): Johnston and Warkentin [18] RE1. Mobile health services work for solving these problems.

RE2. Mobile health services are effective for solving these problems.

RE3. When using mobile health services, solving these problems is more likely to be guaranteed.

Self-Efficacy (SE): Johnston and Warkentin [18]

SE1. It is easy for me to use mobile health services. SE2. I have the capability to use mobile health services.

SE3. I am able to use mobile health services without much effort.

Response Costs (RC): Lee and Larsen[33]
RC1. Mobile health services are expensive to purchase.

RC2. I have to spend effort on learning how to use mobile health services

RC3. Using mobile health services will change my life style.

\section{References}

[1] J. Jiang and A. F. Cameron, "IT-Enabled Self-Monitoring for Chronic Disease Self-Management: An Interdisciplinary Review," MIS Quarterly, vol. Forthcoming, 2019.

[2] A. Savoli, H. Barki, and G. Pare, "Examining How Chronically III Patients' Reactions to, and Effective Use of, Information Technology Can Influence How Well They SelfManage Their Illness," MIS Quarterly, vol. Forthcoming, 2019.

[3] Y. Sun, N. Wang, X. Guo, and Z. Peng, "Understanding the acceptance of mobile health services: a comparison and integration of alternative models," Journal of Electronic Commerce Research, vol. 14, pp. 183-200, 2013.

[4] X. Guo, Y. Sun, N. Wang, Z. Peng, and Z. Yan, "The dark side of elderly acceptance of preventive mobile health services in China," Electronic Markets, vol. 23, pp. 49-61, 2013.

[5] Y. Zhao, Q. Ni, and R. Zhou, "What factors influence the mobile health service adoption? A meta-analysis and the moderating role of age," International Journal of Information Management, vol. 43, pp. 342-350, 2018.

[6] R. W. Rogers, "A protection motivation theory of fear appeals and attitude change," Journal of Psychology, vol. 91, pp. 93-114, 1975.

[7] S. Milne, P. Sheeran, and S. Orbell, "Prediction and intervention in health - related behavior: A meta - analytic review of protection motivation theory," Journal of Applied Social Psychology, vol. 30, pp. 106-143, 2000.

[8] N. D. Weinstein, "Testing four competing theories of health-protective behavior," Health psychology, vol. 12, pp. 324-333, 1993.

[9] R. S. Lazarus and S. Folkman, Stress, appraisal, and coping: Springer publishing company, 1984.

[10] A. Vance, M. Siponen, and S. Pahnila, "Motivating IS security compliance: insights from habit and protection motivation theory," Information \& Management, vol. 49, pp. 190-198, 2012.

[11] C. Posey, T. L. Roberts, and P. B. Lowry, "The impact of organizational commitment on insiders' motivation to protect organizational information assets," Journal of Management Information Systems, vol. 32, pp. 179-214, 2015.

[12] P. C. Fiss, "Building better causal theories: A fuzzy set approach to typologies in organization research," Academy of Management Journal, vol. 54, pp. 393-420, 2011.

[13] V. H. Vroom, Work and Motivation. New York, London, and Sydney: John Wiley and Sons, 1964.

[14] A. Bandura, "Self-Efficacy: Toward a Unifying Theory of Behavioral Change," Psychological Review, vol. 84, pp. 191-215, 1977.

[15] R. W. Rogers, "Cognitive and psychological processes in fear appeals and attitude change: A revised theory of 
protection motivation," in Social psychophysiology: A sourcebook, J. T. Cacioppo and R. E. Petty, Eds., ed New York: Guilford, 1983, pp. 153-176.

[16] W. A. Cram, J. D'Arcy, and J. G. Proudfoot, "Seeing the Forest and the Trees: A Meta-Analysis of the Antecedents to Information Security Policy Compliance," MIS Quarterly, vol. 43, pp. 525-554, 2019.

[17] C. L. Anderson and R. Agarwal, "Practicing safe computing: a multimedia empirical examination of home computer user security behavioral intentions," MIS quarterly, vol. 34, pp. 613-643, 2010.

[18] A. C. Johnston and M. Warkentin, "Fear appeals and information security behaviors: an empirical study," MIS quarterly, vol. 34, pp. 549-566, 2010.

[19] S. Boss, D. Galletta, P. B. Lowry, G. D. Moody, and P. Polak, "What do systems users have to fear? Using fear appeals to engender threats and fear that motivate protective security behaviors," MIS Quarterly, vol. 39, pp. 837-864, 2015.

[20] A. C. Johnston, M. Warkentin, M. McBride, and L. Carter, "Dispositional and situational factors: influences on information security policy violations," European Journal of Information Systems, vol. 25, pp. 231-251, 2016.

[21] P. Menard, G. J. Bott, and R. E. Crossler, "User motivations in protecting information security: Protection motivation theory versus self-determination theory," Journal of Management Information Systems, vol. 34, pp. 1203-1230, 2017.

[22] T. Herath and H. R. Rao, "Protection motivation and deterrence: a framework for security policy compliance in organisations," European Journal of Information Systems, vol. 18, pp. 106-125, 2009

[23] O. A. El Sawy, A. Malhotra, Y. Park, and P. A. Pavlou, "Seeking the configurations of digital ecodynamics: It takes three to tango," Information Systems Research, vol. 21, pp. 835-848, 2010.

[24] Y. Park, O. A. El Sawy, and P. C. Fiss, "The Role of Business Intelligence and Communication Technologies in Organizational Agility: A Configurational Approach," Journal of the Association for Information Systems, vol. 18, pp. 648-686, 2017.

[25] H. Liang and Y. Xue, "Avoidance of information technology threats: a theoretical perspective," MIS Quarterly, vol. 33, pp. 71-90, 2009.

[26] M. Cismaru and A. M. Lavack, "Interaction effects and combinatorial rules governing protection motivation theory variables: A new model," Marketing Theory, vol. 7, pp. 249$270,2007$.

[27] H. Liang and Y. Xue, "Understanding security behaviors in personal computer usage: A threat avoidance perspective," Journal of the association for information systems, vol. 11, pp. 394-413, 2010.

[28] E. H. Das, J. B. De Wit, and W. Stroebe, "Fear appeals motivate acceptance of action recommendations: Evidence for a positive bias in the processing of persuasive messages," Personality and Social Psychology Bulletin, vol. 29, pp. 650664, 2003.

[29] C. Pechmann, G. Zhao, M. E. Goldberg, and E. T. Reibling, "What to convey in antismoking advertisements for adolescents: The use of protection motivation theory to identify effective message themes," Journal of Marketing, vol. 67 , pp. $1-18,2003$.

[30] P. Krishnamurthy, M. Cismaru, and A. Nagpal, "The role of cost and efficacy in persuasiveness of health recommendations," in Society of Consumer Psychology Summer Conference, Honolulu, Hawaii, July, 2004.

[31] Y. Tong, C.-H. Tan, and H.-H. Teo, "Direct and Indirect Information System Use: A Multimethod Exploration of Social Power Antecedents in Healthcare," Information Systems Research, vol. 28, pp. 690-710, 2017.

[32] Y. Xu, Y. Tong, S. S. Liao, G. Zhou, and Y. Yu, "Understanding indirect system use of junior employees in the context of healthcare," Information \& Management, vol. 55, pp. 759-770, 2018.

[33] Y. Lee and K. R. Larsen, "Threat or Coping Appraisal: Determinants of SMB Executives Decision to Adopt AntiMalware Software," European Journal of Information Systems, vol. 18, pp. 177-187, 2009.

[34] R. W. Brislin, "Back-translation for cross-cultural research," Journal of cross-cultural psychology, vol. 1, pp. $185-216,1970$

[35] J. F. Hair, C. M. Ringle, and M. Sarstedt, "PLS-SEM: Indeed a Silver Bullet," The Journal of Marketing Theory and Practice, vol. 19, pp. 139-152, 2011.

[36] C. C. Ragin, "Qualitative comparative analysis using fuzzy sets (fsQCA)," Configurational comparative methods, vol. 51, 2009

[37] C. Fornell and D. F. Larcker, "Evaluating Structural Equation Models with Unobservable Variables and Measurement Error," Journal of Marketing Research, vol. 18, pp. 39-50, 1981.

[38] C. L. Hsu and J. C. C. Lin, "Acceptance of Blog Usage: The Roles of Technology Acceptance, Social Influence and Knowledge Sharing Motivation," Information \& Management, vol. 45, pp. 65-74, 2008.

[39] J. C. Anderson and D. W. Gerbing, "Structural Equation Modeling in Practice: A Review and Recommended TwoStep Approach," Psychological Bulletin, vol. 103, pp. 411$423,1988$.

[40] J. J. Jiang, G. Klein, and C. L. Carr, "Measuring Information System Service Quality: SERVQUAL from the Other Side," MIS Quarterly, vol. 26, pp. 145-166, 2002.

[41] B. Furneaux and M. Wade, "Impediments to information systems replacement: a calculus of discontinuance," Journal of Management Information Systems, vol. 34, pp. 902-932, 2017. 